\title{
A combined robotic and cognitive training for locomotor rehabilitation: evidences of cerebral functional reorganization in two chronic traumatic brain injured patients
}

\section{Katiuscia Sacco 1,2,3*, Franco Cauda 1,2, Federico D'Agata ${ }^{1,2}$, Sergio Duca ${ }^{2}$, Marina Zettin ${ }^{4}$, Roberta Virgilio4, Alberto Nascimbeni ${ }^{5}$, Guido Belforte ${ }^{6}$, Gabriella Eula ${ }^{6}$, Laura Gastaldi ${ }^{6}$, Silvia Appendino ${ }^{6}$ and Giuliano Geminiani ${ }^{1,2,3}$}

${ }^{1}$ Department of Psychology, University of Turin, Turin, Italy

${ }^{2}$ Department of Neuroradiology, Koelliker Hospital, Turin, Italy

${ }^{3}$ Neuroscience Institute of Turin, Turin, Italy

${ }^{4}$ Centro Puzzle, Turin, Italy

${ }^{5}$ Physiatric Unit, Santa Croce Hospital, Turin, Italy

${ }^{6}$ Department of Mechanics, Polytechnic of Turin, Turin, Italy

\section{Edited by:}

Julia Karbach, Saarland University,

Germany

Reviewed by:

David Field, University of Reading, UK

Carmen Krewer, Schoen Klinik Bad

Aibling, Germany

\section{*Correspondence:}

Katiuscia Sacco, Department of

Psychology, via Po 14, Turin, Italy

e-mail: katiuscia.sacco@unito.it
It has been demonstrated that automated locomotor training can improve walking capabilities in spinal cord-injured subjects but its effectiveness on brain damaged patients has not been well established. A possible explanation of the discordant results on the efficacy of robotic training in patients with cerebral lesions could be that these patients, besides stimulation of physiological motor patterns through passive leg movements, also need to train the cognitive aspects of motor control. Indeed, another way to stimulate cerebral motor areas in paretic patients is to use the cognitive function of motor imagery. A promising possibility is thus to combine sensorimotor training with the use of motor imagery. The aim of this paper is to assess changes in brain activations after a combined sensorimotor and cognitive training for gait rehabilitation. The protocol consisted of the integrated use of a robotic gait orthosis prototype with locomotor imagery tasks. Assessment was conducted on two patients with chronic traumatic brain injury and major gait impairments, using functional magnetic resonance imaging. Physiatric functional scales were used to assess clinical outcomes. Results showed greater activation post-training in the sensorimotor and supplementary motor cortices, as well as enhanced functional connectivity within the motor network. Improvements in balance and, to a lesser extent, in gait outcomes were also found.

Keywords: motor training, cognitive training, motor imagery, locomotor rehabilitation, brain injury, robotic gait orthosis, functional magnetic resonance imaging, brain plasticity

\section{INTRODUCTION}

Patients with severe traumatic brain injury (TBI) may develop seriously disabling motor disorders, which may be due to lesions of the corticospinal pathways, and extrapyramidal as well as multisensory dysfunction. Deconditioning also decreases somatosensory input and disrupts body image. Rehabilitation exercises that stimulate the remaining, intact central nervous system are based on the assumption that the brain partly makes up for lost functions, through neuroplasticity. During the learning of new skills, cortical regions associated with sensorimotor function of the body parts most utilized for the skilled task gradually start to be represented over larger cortical territories (Pascual-Leone et al., 1994; Karni et al., 1995). Besides, some studies have shown that functional and structural changes take place in the cerebral cortex after injury (for a review, see Rossini et al., 2007). These two modulators of cerebral function, behavioral experience and brain injury, interact. Hence it is likely that after traumatic brain injuries, the sensorimotor experiences of the individual can remodel the structure and function of undamaged parts of the brain, thus promoting recovery.

The most recent technique for gait rehabilitation makes use of robotic systems that move the patient's legs in a physiological way on a moving treadmill, while a body weight support (BWS) system with its harness supports the patient's weight. It has been demonstrated that automated locomotor training can improve walking capabilities in spinal cord-injured subjects (Colombo et al., 2000, 2001; Jezernik et al., 2003; Wirz et al., 2005), but its effectiveness on brain damaged patients - i.e., stroke and TBI patients - has not been well established. Indeed, while some studies on stroke patients found better outcomes when robotic rather than conventional training is used (e.g., Mayr et al., 2007; Schwartz et al., 2009), other studies (e.g., Husemann et al., 2007; Westlake and Patten, 2009) obtained intermediate results (found no significant differences in primary outcomes between conventional and 
robotic therapy, but more improvements on secondary outcomes in the robotic-assisted group), and some others even found the opposite result, i.e., superior effectiveness for the conventional training (e.g., Hornby et al., 2008; Hidler et al., 2009), and thus cast doubts on the validity of robotic- vs. therapist-assisted locomotor training in chronic post-stroke patients. A Cochrane review (Mehrholz et al., 2007) on 414 stroke patients showed that robotic training in combination with physiotherapy improves some gait parameters, but not others; it also suggests caution in interpreting the results, because protocols and patient status vary greatly across studies and some trials tested electromechanical devices in combination with functional electrical stimulation. Finally, as far as TBI patients are concerned, we found no study employing robotic gait rehabilitation (RGR) protocols. A possible explanation of the above mentioned discordant results on the efficacy of robotic training in patients with cerebral lesions could be that these patients, besides stimulation of physiological motor patterns through passive leg movements, also need to train the cognitive aspects of motor control. Indeed, another way to stimulate cerebral motor areas in paretic patients is to use the cognitive function of motor imagery, which implies that the subject forms a representation of a given motor act: during kinesthetic motor imagery the subject is asked to imagine the introspective sensorimotor feeling of moving the limb. There are evidences that brain injured patients retain the ability to generate accurate motor images of actions they cannot perform (Decety and Boisson, 1990; Sirigu et al., 1995), and that mental practice of motor skills can improve actual performance (Jackson et al., 2001). Thus, motor imagery can be considered a potentially effective intervention in the rehabilitation of patients with motor impairments. However, the efficacy of this technique could be limited by the fact that imagery does not provide somatosensory afferents, which constitute the main intrinsic feedback in relearning movements. A promising possibility is thus to combine sensorimotor training with the use of motor imagery (Jackson et al., 2004; Malouin et al., 2004).

A combined robotic and cognitive protocol for locomotor rehabilitation had not been developed so far. We therefore designed a robotic and cognitive gait rehabilitation (RCGR) protocol, whose strength lies in the integrated use of both sensorimotor and cognitive stimulations. Sensorimotor training is provided thanks to a pneumatic active gait orthosis that we designed and built (Belforte et al., 1997, 2001), which induces lower limb movements. The robotic orthosis design and its characteristics are briefly described in this paper. Cognitive training consists of a series of locomotor imagery tasks to be performed both during and immediately after the robotic-assisted session. The proprioceptive and kinesthetic activation induced by the passive leg movements provides reproducible and constant afferent input to the motor control centers, facilitating central pattern generators and enhancing motor drive; also, such proprioceptive sensations are essential for the parallel cognitive training. Indeed, it is very difficult to imagine a procedural action such as that of walking, as it normally does not require any conscious attention. Thus, the proprioceptive inputs received during the passive training are the only help the patient has when mentally representing a motor sequence of locomotion. On the other hand, the mental imagery employed during the robotic-assisted motion focuses the patient's conscious attention on the ongoing steps: as walking in normal subjects is an automated - mainly subcortical - activity, focusing the patient's conscious attention on the movements involved in ambulation is crucial in order to make him/her reacquire motor representations.

The goal of this paper is to evaluate the brain changes following our RCGR protocol by evaluating possible cerebral functional reorganizations. To this end, we submitted two clinical cases (chronic paretic patients with TBI) to our RCGR protocol and assessed their cerebral changes using functional magnetic resonance imaging (fMRI), an in vivo imaging technique which allows the mapping of active processes within the brain. fMRI has been previously used to study training-induced plasticity in stroke patients (for a review see Nelles, 2004); locomotor trainingrelated brain changes have been recently investigated in children with cerebral lesions (de Bode et al., 2007; Phillips et al., 2007), but similar data for adult patients is still lacking. To the best of our knowledge, there are no fMRI studies assessing RGR in adult brain injured patients.

The fMRI assessments were aimed at investigating whether the RCGR rehabilitation led to changes in cerebral activations. Our predictions are based on the results of previous work we carried out on healthy subjects (Sacco et al., 2006, 2009): we found that combined locomotor and cognitive training modifies sensorimotor activation of the brain, leading to greater activation of the premotor and supplementary motor areas (SMA), the primary motor and somatosensory areas of the dominant hemisphere, as well as an increasing functional connectivity within the motor network. A manifestation of functional connectivity is the covariance of metabolic rates in functionally related brain regions (Friston et al., 1993): coherent changes in blood flow imply neuronal connections. Thus, in line with our previous results, we hypothesized that the RCGR training can enhance both sensorimotor activations in the cortical areas involved in lower limb representation, and functional connectivity, i.e., interconnections between brain regions.

\section{MATERIALS AND METHODS THE ROBOTIC GAIT ORTHOSIS}

In the last decade, many robotic devices for lower limb rehabilitations have been developed (for a recent review see Waldner et al., 2009). The robotic gait orthosis we used is a prototype developed by our group for TBI gait rehabilitation purposes (differences from the existing devices are described below). It consists of a modified reciprocating gait orthosis (RGO) integrated with a pneumatic actuation system for knee and hip joints. Hinges that enable rotation in the sagittal plane replace the RGO's original locked joints. The hip angle ranges from $-20^{\circ}$ flexion to $20^{\circ}$ extension and the knee angle from $0^{\circ}$ extension to $90^{\circ}$ flexion. Joint actuation is provided by double acting pneumatic cylinders that are positioned on the passive RGO structure and controlled by a PLC (Programmable Logic Controller) and a group of electrovalves. See Figure 1.

For the hip actuation (Figure 2A), a cylindrical tube is fixed to the rear RGO tube, whereas the rod is hinged on a metal plate that is integral with the femoral segment of the orthosis. A cable connects the two hips and makes their movement reciprocal, that is the extension of one hip achieves flexion in the opposite hip. This enables a crossed hip joint actuation strategy and a simple 


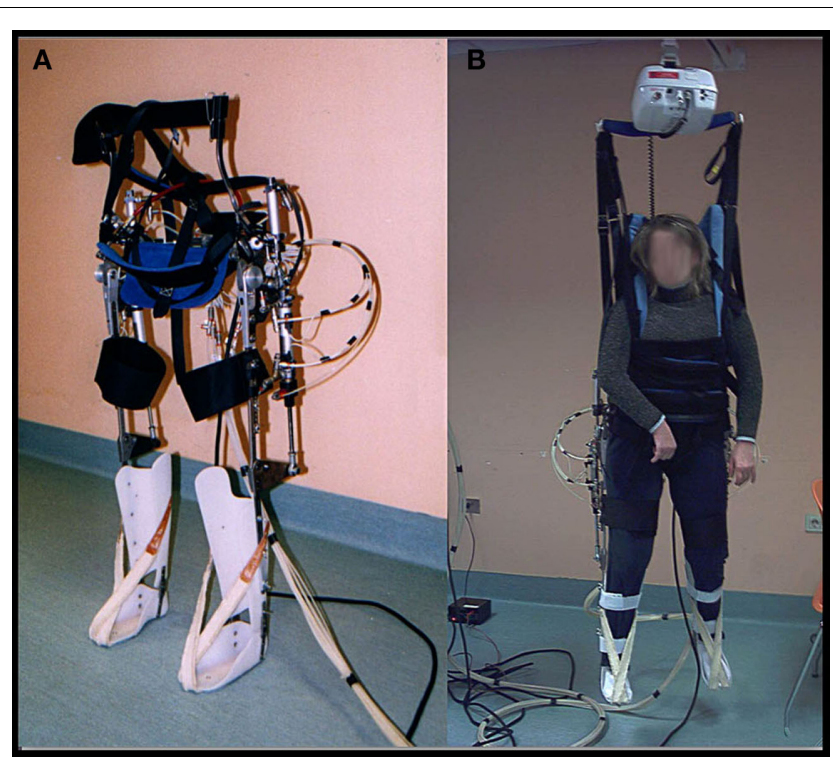

FIGURE 1 | Active RGO used for training, alone (A) and worn by subject (B).

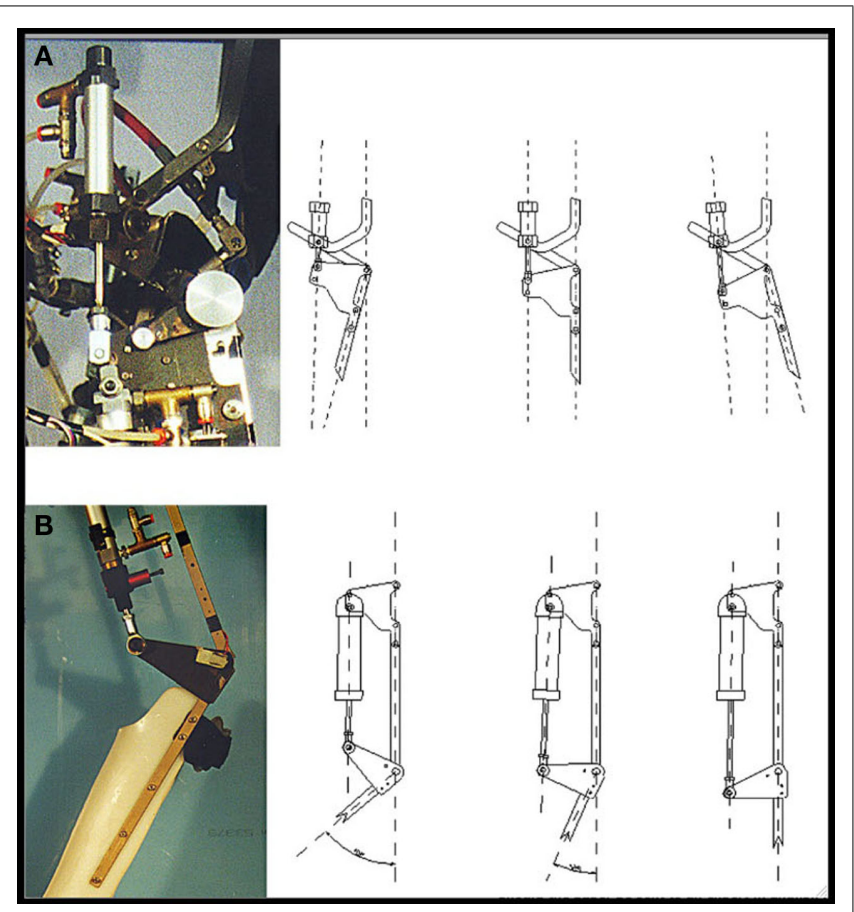

FIGURE 2 | Details of hip (A) and knee (B) actuation system. (Source, Li et al., 2008).

control of the neutral trunk position with respect to the legs. Knee actuation (Figure 2B) is based on the same principle: a double acting pneumatic cylinder has its tube fixed to the orthosis's femoral segment whereas its rod is hinged on a metal plate that is integral with the tibial segment. The suitably positioned hinge helps create the necessary lever arm action to generate appropriate torque on the joint. The ankle joint is passive: the foot and calf are fixed to an ankle foot orthosis (AFO) and elastic elements keep the foot in slight dorsiflexion so to prevent it dropping. The robotic orthosis is able to reproduce a gait cycle according to two different modes: step-by-step or continuous. Step-by-step mode requires user consent after each step in order to proceed to the following step, whereas in the continuous mode the orthosis imposes the gait cycle according to established parameters.

The robotic orthosis is used together with a BWS system, as in most rehabilitation protocols, while, unlike other devices, it does not make use of a treadmill. This choice has been made to allow the patient actually to move forward, according to the kinematic settings. This system should offer a more physiological context for gait rehabilitation, avoiding the proprioceptive, visual, and vestibular mismatch generated by walking on the spot on a treadmill. Moreover, patients with TBI often exhibit not only pyramidal motor impairment but also major balance and coordination disorders, owing to multi-level cerebellar, vestibular, and sensorial damage, thus generating additional difficulties in carrying out functional dynamic tasks (Basford et al., 2003). A more physiological sensorimotor task would also enable more coherent perception (Berthoz, 2000), enhancing memory of movement and facilitating motor imagery sessions. Finally, a gait system not bound to a treadmill allows training in different kinds of environments, such as slopes, steps, etc., without requiring large spaces or structured environments: a room with a ceiling guide for simple BWS is sufficient.

Our robotic system, unlike the existing devices, uses a pneumatic as opposed to an electric actuation system. This choice has been made primarily because compressed air is very compliant and thus helps in avoiding clasp-knife rigidity, i.e., a sudden increase in tone when antigravity muscles are contracted. Indeed, this phenomenon, which is due to spasticity and thus very frequent in brain injured patients, abnormally increases resistance on passive stretching and interferes with both extension and flexion. Also, compressed air is intrinsically safe, clean, and usually easily available in most medical centers.

\section{PATIENTS AND REHABILITATION PROTOCOL}

The protocol was approved by the local Ethics Committee (Department of Psychology, University of Turin, Italy). Two chronic traumatic brain injured patients were recruited from the "Centro Puzzle" in Turin. The patients gave their written informed consent for both the rehabilitation protocol and the fMRI scanning. The inclusion criteria required an observable walking deficit, active ankle dorsi- and plantarflexion, and no observable motor recovery in the previous 12 months in spite of standard rehabilitation programs. The exclusion criteria comprised the presence of lower limb peripheral neuropathies, spinal lesions, previous pathologies of the central nervous system, cognitive deterioration (MMSE $<24$ ), aphasia, psychiatric illness or severe behavioral alterations, drug or alcohol abuse, severe visual or auditory deficits, severe orthopedic impairments, and magnetic resonance incompatible intra-body devices.

Patient S.R., was a 28-year-old male, right-handed and rightfooted, with TBI that had occurred 5 years earlier, with diffuse axonal damage and major gait impairment owing to cerebellar 
ataxia which also hindered his ability to stand but not his head and trunk control while sitting: mild dysmetria was present in the four limbs. There were no clinical signs of spasticity (Ashworth Scale grade $0 / 4$, meaning no increase in muscle tone), although mild hyperreflexia was present in the right upper limb. Muscle strength was preserved [Medical Research Council (MRC) grade 5/5 for both upper and lower extremities, meaning normal power]. Sensibility, position sense and kinesthesia were undisturbed.

Patient M.E., was a 24-year-old female, right-handed and rightfooted, with TBI that had occurred 2 years earlier, with diffuse axonal injury, severe tetra-paresis and heavily impaired gait. Standing was possible only with feet apart and for less than $30 \mathrm{~s}$. Trunk and head control was good while sitting. There were signs of spasticity at the right lower limb and the patient had an equinus foot, albeit without signs of contracture (Ashworth scale grade for each limb; mean of the three segments of each limb): 3.3 right inferior limb; 2 right superior limb; 2 left inferior limb; 1 left superior limb). Muscle strength was reduced such that the joint can be moved only against gravity with the examiner's resistance completely removed (MRC grade 3/5 for both upper and lower extremities). A mild hypoesthesia was detected in the four limbs.

The patients underwent our RCGR protocol, which comprised three sessions per week over a 4-week period; each session lasted $20 \mathrm{~min}$. Treatment frequency was based on existing locomotor imagery practice protocols (e.g., Dickstein et al., 2004; Dunsky et al., 2006); session duration was based on recommendations to limit motor imagery sessions to $20 \mathrm{~min}$, as there is a negative relationship between effect and increased practice duration (Driskell et al., 1994). As there are no standardized guidelines for clinical motor imagery protocols, we used the existing studies on locomotor imagery practice (summarized in Malouin and Richards, 2010) to inform our protocol. The instructions were mainly oriented towards the kinesthetic, rather than the visual, aspects of the task, in order to focus the patients' attention on the proprioceptive inputs given by the robot. In the first $10 \mathrm{~min}$, the patient was supported by means of the BWS system, while the robotic orthosis - set to the continuous mode-moved his/her legs, reproducing rhythmical walking patterns. The hip range of motion was $40^{\circ}$ $\left(20^{\circ}\right.$ extension and $20^{\circ}$ flexion $)$, whereas the knee range of motion was from extended knee to $60^{\circ}$ flexion. During robotic gait, the therapist, in enabling the robot progression, asked the patients to mentally perform cognitive tasks aimed at focusing their conscious attention on the ongoing steps, feeling proprioceptive and kinesthetic inputs, and thinking of the mental actions needed for the mental reproduction of a movement. In order to cognitively engage the patient, the therapist stopped the robot at pre-defined time points and asked the patient to describe the position of his/her hips, knees, and feet, without looking at them ${ }^{1}$; afterwards, starting from that position, the patient had to imagine making some other steps, following a metronome, and then, at a random metronome

\footnotetext{
${ }^{1}$ Acceptable descriptions were like the following: "My right leg is ahead, with my foot flat, my knee bent, and my hip forward, while my left leg is behind, as if I was to finish a step with my right leg." Despite most of the times patients needed to be prompted by therapist's questions before producing a complete description, they showed to be good at this task, as their descriptions matched their actual legs' position in every trial.
}

stop, the patient had to describe the imagined final position of his/her limbs. During the following 10 min the patient, still with BWS but without the robotic orthosis, was placed on a platform equipped with parallel bars that (s)he could hold: there (s)he was asked to recall the kinesthetic feelings of the preceding phase and to use them to perform locomotor imagery-related tasks in the first person perspective, involving different conditions such as standing, initiating gait, walking, and walking with obstacles. At the end of this phase, the patient was asked to walk along the platform, whilst continuing to concentrate on his/her body as it moved. The RCGR protocol was administered by one of the authors of the present paper (RV), a clinical neuropsychologist working at the Centro Puzzle in Turin. During the training period, patients received their standard physiotherapy.

As clinical measures we selected: the standing balance scale (SBS; Bohannon, 1989) for balance evaluation, the Massachusetts General Hospital Functional Ambulation Classification (FAC; Holden et al., 1986) for gait function, and the Barthel Index (BI; Mahoney and Barthel, 1965) for assessment of assistance need in activities of daily living. Assessments were carried out before and after the RCGR protocol. Outcome measures were administered by an independent rater, i.e., a physiatrist who was blind with respect to the treatment applied to the patient and his/her participation in an experimental rehabilitation protocol.

\section{fMRI PROCEDURES}

In order to define the brain correlates of locomotion using fMRI, a specific task implying extension, and flexion of the ankle joint has been proposed in the current literature (Dobkin et al., 2004); its validity has been demonstrated by experimental work showing that foot extension and flexion alone generate a similar brain activation pattern to that associated with walking (see for example Sahyoun et al., 2004). Indeed, movements of other lower limb joints, such as the knee or hip, are problematic in fMRI studies, as they propagate through the vertical body plane, causing head motion. Consequently, ankle plantar- and dorsiflexion represents the gold standard fMRI paradigm for gait analysis, and thus we adopted it in our study.

During fMRI, the patient was required to perform plantarflexion (downward) and dorsiflexion (upward). At the beginning of each scanning session, patients were individually instructed on the task they were going to perform during scanning. The experimenter showed the stimuli, as well as the type, amplitude and speed of the movements required; the subject was asked to perform each movement for a few seconds. The task was performed using a block design with $12 \mathrm{~s}$ of rest alternating with $12 \mathrm{~s}$ of the active condition. In the active condition, subjects moved their right foot and left foot alternately. In the rest condition, they had to relax, without performing any movements. Movements were performed at $0.5 \mathrm{~Hz}$, as this rate is similar to that of ankle movements during walking. As far the movement amplitude is concerned, the patient was asked to perform the maximal plantarflexion and then a dorsiflexion of about $20-30^{\circ}$ and come back to the starting position in plantarflexion. Subjects performed the task with their shoes off and their legs slightly raised and supported by pillows. Sandbags were placed on both legs in order to limit leg movements. The stimuli were visual and represented two feet. Both feet were white 
in the rest condition, the right foot turned red and the left foot remained white when the subject had to move their right foot, and vice-versa when the subject had to move their left foot. The task lasted $5 \mathrm{~min}$. It was generated using the E-Prime software (Psychology Software Tools, Inc., Pittsburgh, PA, USA). A color LCD screen projected the visual stimuli onto a rear-projection screen in the bore of the magnet. The participants viewed this screen via an angled mirror system. The stimuli were presented by IFIS$\mathrm{SA}^{\mathrm{TM}}$ (MRI Device Corporation, Waukesha, WI, USA), which also synchronized the presentation of stimuli with the fMRI scanner.

Data acquisition was performed on a $1.5 \mathrm{~T}$ Intera scanner (Philips Medical Systems). Functional $\mathrm{T}_{2}$-weighted images were acquired using echoplanar (EPI) sequences, with a repetition time (TR) of $3000 \mathrm{~ms}$, an echo time (TE) of $60 \mathrm{~ms}$, and a $90^{\circ}$ flip angle. The acquisition matrix was $64 \times 64$; the field of view $(\mathrm{FoV})$ was $256 \mathrm{~mm}$. For each task, a total of 100 volumes were acquired. Each volume consisted of 25 axial slices, parallel to the anteriorposterior (AC-PC) commissure line and covering the whole brain; the slice thickness was $4 \mathrm{~mm}$ with a $0.5-\mathrm{mm}$ gap. Two scans were added at the beginning of functional scanning and the data discarded to reach a steady state magnetization before acquisition of the experimental data. In the same session, a set of threedimensional high-resolution $\mathrm{T}_{1}$-weighted structural images was acquired for each participant. This data set was acquired using a fast field echo (FFE) sequence, with a repetition time (TR) of $25 \mathrm{~ms}$, the shortest echo time (TE), and a $30^{\circ} \mathrm{flip}$ angle. The acquisition matrix was $256 \times 256$; the FoV was $256 \mathrm{~mm}$. The set consisted of 160 sagittal contiguous images covering the whole brain. The in-plane resolution was $1 \mathrm{~mm} \times 1 \mathrm{~mm}$ and the slice thickness was $1 \mathrm{~mm}(1 \mathrm{~mm} \times 1 \mathrm{~mm} \times 1 \mathrm{~mm}$ voxels $)$.

We analyzed imaging data using Brain Voyager QX (Brain Innovation, Maastricht, the Netherlands). The functional data of each subject underwent the following preprocessing steps: mean intensity adjustment, head motion correction, slice scan time correction, spatial data smoothing [full width at half maximum $(\mathrm{FWHM})=4 \mathrm{~mm}]$, temporal filtering, and temporal smoothing $(\mathrm{FWHM}=2.8 \mathrm{~s})$. After preprocessing, each subject's slice-based functional scans were coregistered to their 3D high-resolution structural scan, and the 3D structural data set of each subject was transformed into Talairach space (Talairach and Tournoux, 1988). Using the anatomical-functional coregistration matrix and the determined Talairach reference points, we transformed the functional time course of each subject into Talairach space and created the volume time course. For each patient, a single-subject study design matrix was specified and the defined box-car was convolved with a pre-defined hemodynamic response function (HRF) to account for the hemodynamic delay. A statistical analysis using the general linear model was performed to yield functional activation maps during the pre- and post-tests separately. Subsequently, the general linear model was use to compare post-test activations with pre-test activations for each patient. All statistical comparisons were computed at a statistical threshold of $p<0.05$, corrected for multiple comparisons using Bonferroni correction. We measured functional connectivity using the seed voxel method. For each patient, we selected a cluster of 10 contiguous seed voxels within the SMA of the left (dominant) hemisphere. The cluster seed included the voxel with the most task-related activity in the foot task, and significant voxels surrounding it. Time courses at each voxel of the seed cluster were averaged. Next, the time course for the seed voxel cluster was correlated with every other voxel time course in the brain. Nuisance factors were used as covariates; they included head movements in the six directions and a 50-voxel region of interest (ROI) in the cerebrospinal fluid. Voxel time courses correlating significantly $(p<0.05$ corrected for multiple comparisons using Bonferroni correction) were considered to be functionally connected. To identify changes in connectivity between the pre- and post-test conditions, a $t$-test was applied on the pre- and post-test connectivity maps to determine regions with significantly different connectivity across conditions $(p<0.05$ corrected for multiple comparisons using the Bonferroni correction).

\section{RESULTS \\ CLINICAL OUTCOMES}

Both patients carried out the overall program and no complications were recorded; M.E. did not complete some training sessions (i.e., in the first three sessions the patient completed the first $10 \mathrm{~min}$ with the robotic orthosis, but was unable to complete the second part of the training session with parallel bars) owing to a lack of postural comfort. No change in spasticity or strength were observed.

\section{PATIENT S.R.}

On the SBS the income measure was $2 / 10$, which implies standing for $30 \mathrm{~s}$. with feet apart, and the outcome measure was 3/10, which implies standing with feet in contact for less than $30 \mathrm{~s}$. On the FAC, the income measure was $1 / 6$, meaning the absence of functional ambulation (he ambulated in parallel bars only), and the outcome measure was $2 / 6$, meaning the ability to walk $10 \mathrm{ft}$ or more outside parallel bars (requiring continuous manual contact of one person). On the BI, the income measure was 55/100, and the outcome measure was 70/100. This increment of 15 points resulted entirely from improvements on all the postural/gait related items of the scale, i.e., transfers (bed to chair and back), mobility (on level surfaces), and stairs.

In summary, S.R. improved on all scales used, and these improvements were clinically significant: SBS indicated a progress from standing with feet apart to standing with feet in contact; FAC indicated a progress from non-functional ambulation to ambulation; BI indicated progresses from 25 to $50 \%$ on each gait item. Thus, after the treatment, S.R. improved both balance and gait, being able to walk outside the parallel bars.

\section{PATIENT M.E.}

On the SBS the income measure was $1 / 10$, which implies inability to stand even with feet apart, and the outcome measure was $2 / 10$, which implies standing for at least $30 \mathrm{~s}$ with feet apart. On the FAC, the income measure was $1 / 6$, meaning the absence of functional ambulation, and the outcome measure remained unaltered. Nonetheless, while at income M.E. could not ambulate at all, at the outcome she could ambulate in parallel bars. Even if this progress cannot be detected on the physiatric scale, it is indeed clinically relevant, as it makes the patient able to do rehabilitation exercises that she was unable to perform before, with the aim of possibly 
walking with support. Also on the BI, income and outcome measures were the same, as the progresses of M.E. fell in between score categories and thus produced no detectable improvement.

In summary, M.E. showed an improvement in balance, with decreased back sway, and more subtle progresses in ambulation, not detectable by the physiatric scales which we used.

\section{fMRI RESULTS}

Both patients managed to perform the fMRI task. In both patients, pre-RCGR fMRI testing showed activations in the foot and leg primary motor area (M1) and in the SMA.
In both patients, comparing the spatial distribution of patterns of brain activation pre- and post-RCGR revealed extended bilateral activations in the SMA, as well as activations in the cingulate motor cortex, and in the foot somatosensory motor area (S1). In patient S.R. activations in the cerebellum also emerged. Figure 3 shows pre- and post-training activations on a sagittal view, for each patient. Figure 4 represents three-dimensional cortex reconstructions of the dominant hemisphere, for each patient: "green" indicates activations in the pre-training condition; "yellow" indicates activations in the post-training condition. All statistical comparisons were computed at a statistical threshold of

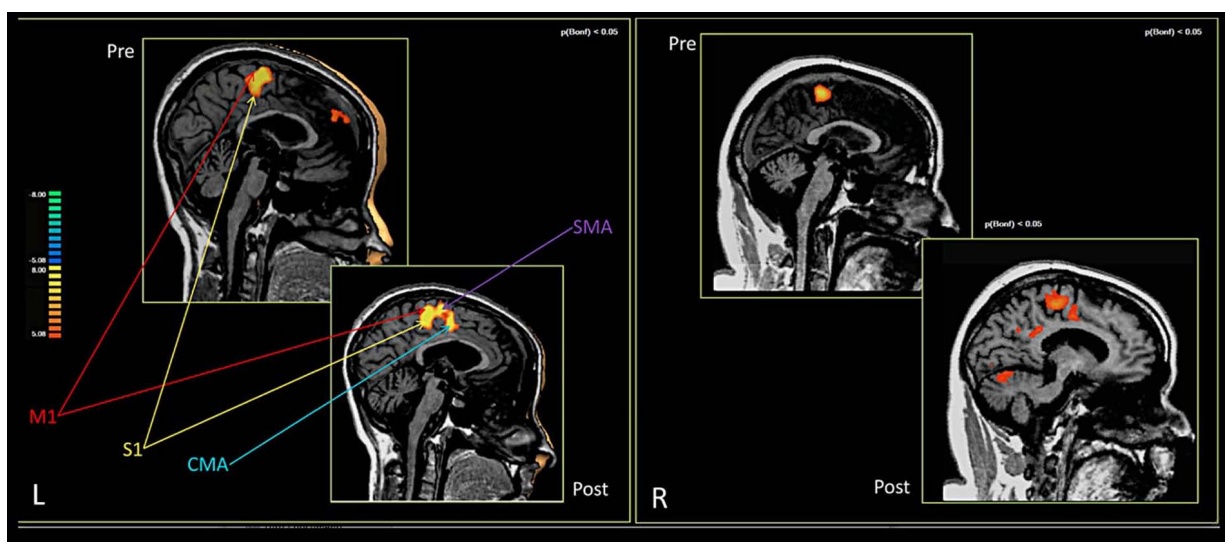

FIGURE 3 | Brain activations in the pre- and post-training conditions. Patient M.E. on the left. Patient S.R. on the right.

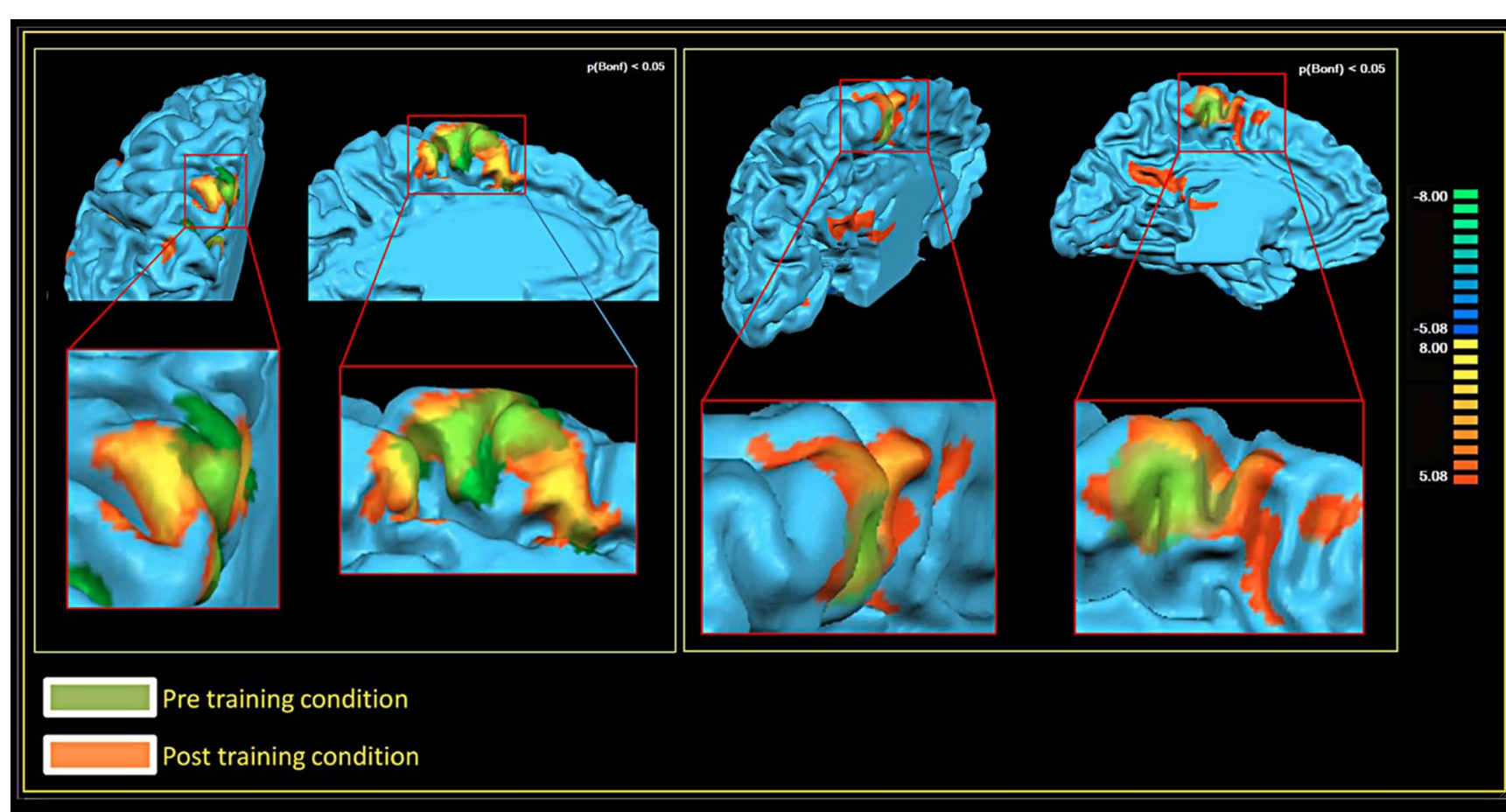

FIGURE 4 | Brain activations in the pre- and post-training conditions, 3D cortex reconstruction of the left hemisphere. Patient M.E. on the left. Patient S.R. on the right. 
$p<0.05$, corrected for multiple comparisons using Bonferroni correction.

As far as functional connectivity is concerned, it could not be computed on patient M.E. due to technical problems. It was, however, possible for S.R.: here, the seed voxel correlation analysis demonstrated an increase in functional connectivity. Figure 5 shows the pre- and post-connectivity patterns of S. R.: "yellow" indicates connectivity in the pre-training condition; "blue" indicates connectivity in the post-training condition. All statistical comparisons were computed at a statistical threshold of $p<0.05$, corrected for multiple comparisons using Bonferroni correction.

\section{DISCUSSION}

As we reported in the introduction, the use of RGR in subjects with brain lesions is still controversial. Indeed, these patients show gait disruption caused not only by damage to motor pathways but also by impairments of perception, attention, and body schema. For these reasons, it is important to understand the brain mechanisms leading to gait improvements. To this end, neuroimaging techniques can help in investigating the possible cerebral changes taking place during the treatment. Greater fMRI activation of cortical sensorimotor areas after RGR in incomplete spinal cordinjured patients (Winchester et al., 2005) and increased corticomotor excitability after treadmill training in chronic stroke patients (Yen et al., 2008) have been demonstrated; however, evidence of neuroplasticity after RGR in chronic brain injured patients was still lacking.

Besides, the published works investigating RGR in brain injured patients involved only stroke participants. Studies on TBI patients are lacking in the literature probably because such patients are heterogeneous in their clinical representation, usually presenting diffuse axonal damage and a focal lesion; it is therefore not easy to compare the effects across subjects. Also, unlike stroke patients in whom the gait problem is hemi-paresis/plegia, TBI patients often present para- or tetra-paresis/plegia, and this renders the therapy more complex. However, most of these patients are young and thus the treatment can benefit from a greater brain plasticity, and an efficacious rehabilitation leads to significant individual and social effects.
The present paper presented two case reports - TBI patients with major gait impairments - studied with fMRI before and after a robotic locomotor rehabilitation. Our protocol made use of a newly developed gait system specifically designed to train brain injured patients: its pneumatic actuations are intended to counter spasticity, and the absence of a treadmill should help to train patients in a more coherent perceptual framework. Together with robotic assistance, we employed motor imagery as a complementary technique. The proposed RCGR protocol should improve gait by facilitating central pattern generators and also by enhancing cognitive aspects of motor relearning. These processes should stimulate cortical neuroplasticity, investigated by fMRI.

Our neuroimaging results supported our hypotheses, showing greater activations post-training in the sensorimotor and supplementary motor cortices, as well as enhanced functional connectivity within the motor network. Such results are in line with the previous studies that we have carried out on healthy subjects (Sacco et al., 2006, 2009), as well as with the literature on motor training in normal and pathological subjects (for reviews, see Kelly et al., 2006; Rossini et al., 2007; Enzinger et al., 2008; Forrester et al., 2008). Besides, similar changes in sensorimotor and supplementary motor cortices through locomotor exercises have been associated with improved gait function in neurologically impaired children and adults (de Bode et al., 2007). Finally, the augmented connectivity, being a manifestation of the covariance of metabolic rates in functionally related brain regions, suggests a reinforcement of the strength of existing synapses.

At a clinical level, the main result we obtained was an improvement of balance, which was evident in both patients. Balance plays a major role in posture maintenance, and it is a prerequisite for ambulation; most postures demand constant sustained activity implying tone activation of muscles, designated as the tonic component of voluntary movements. It has been shown that kinetic imagery is associated with an increase of both the muscle tone and the excitability of the corticospinal pathway (Milton et al., 2008). Observations following hemispherectomy in both primates (Lawrence and Kuypers, 1968) and humans (de Bode et al., 2005) suggest that, while the phasic component of voluntary

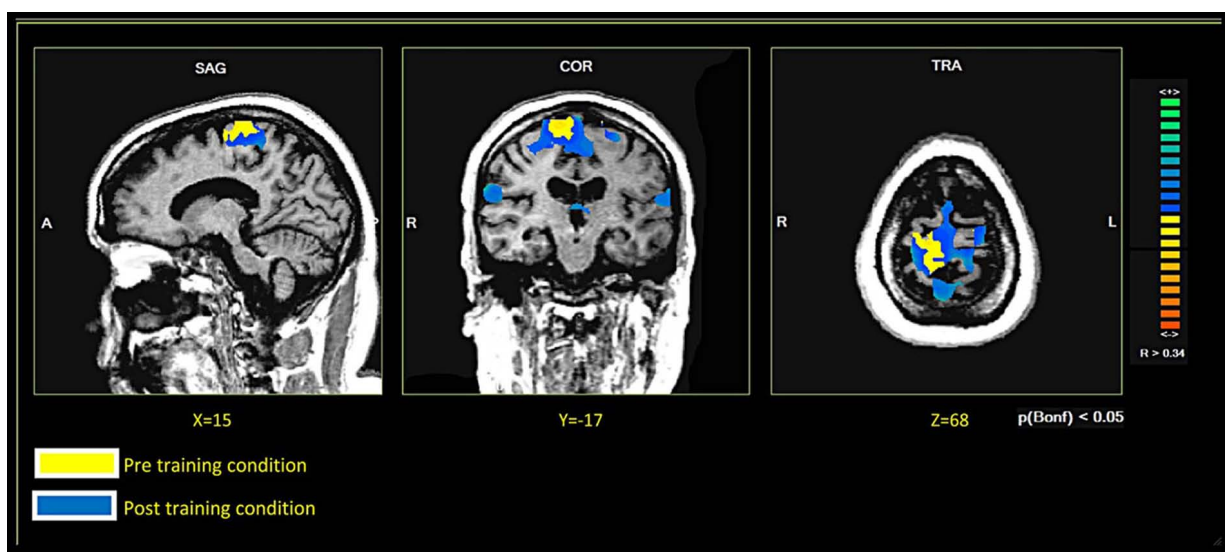

FIGURE 5 | Brain connectivity in the pre- and post-training conditions: patient S.R. 
movements is most closely associated with activation in M1, the tonic component is most likely associated with activation in other cortical and subcortical regions. Kinetic motor imagery activates regions of the cortex prevalently involved in the control of the tonic components of movement (Milton et al., 2008). Thus, it is plausible that the motor imagery components of our protocol helped in improving balance. The increased post-training activations we found in the SMA and S1, together with those of the cerebellum, are consistent with such an interpretation.

As far as gait outcomes are concerned, while one patient (S.R.) showed significant improvements on all clinical scales, in the other patient (M.E.) progresses in ambulation were clinically evident but more subtle and not detectable by the physiatric rating scales we used. However, for chronic TBI subjects with major motor impairments, even minor improvements might have positive effects on the perceived quality of life. Besides, the functional neuroimaging modifications, which were also observed in patient S.R., suggest that brain mechanisms are liable to changes, which may require a greater amount of time and training to be converted to behavioral, detectable outcomes.

Finally, the robotic orthosis we developed, despite the limitations indicated below, was shown to be suitable for this kind of patient. Indeed, it could also be used with the active participation of patients, exploiting their remaining gait capacity; as active participation stimulates motor recovery, future work with this orthosis should also involve less severe TBI patients, in order to investigate the effect of the patient's active contribution.

In conclusion, our RCGR protocol appears to be a useful tool for gait rehabilitation in TBI patients, whose primary impact is on balance impairment. It may enhance both the subcortical motor automatisms and the cortical processes of motor learning.

\section{REFERENCES}

Basford, J. R., Chou, L. S., Kaufman, K. R., Brey, R. H., Walker, A., Malec, J. F., Moessner, A. M., and Brown, A. W. (2003). An assessment of gait and balance deficits after traumatic brain injury. Arch. Phys. Med. Rehabil. 84, 343-349.

Belforte, G., Gastaldi, L., and Sorli, M. (1997). "Active orthosis for rehabilitation and passive exercise," in Proceedings of the International Conference on Simulations in Biomedicine, Biomed, 199-208.

Belforte, G., Gastaldi, L., and Sorli, M. (2001). Pneumatic active gait orthosis. Mechatronics 11, 301-323.

Berthoz, A. (2000). The Brain's Sense of Movement. Cambridge, MA, London: Harvard University Press

Bohannon, R. W. (1989). Correlation of lower limb strengths and other variables with standing performance in stroke patients. Physiother. Can. 41, 198-202.

Colombo, G., Joerg, M., Schreier, R., and Dietz, V. (2000). Treadmill training of paraplegic patients using a

Systematic studies involving a greater number of participants and follow-up assessments are necessary in order to confirm our suggestions.

\section{LIMITATIONS}

This study is of an exploratory nature, being limited to the observation of only two patients: it demonstrates that the RCGR program can be effective for some TBI patients, but it provides no information about what proportion of such patients will benefit from its use. Thus, further systematic research is needed to address clinical outcomes. Moreover, as our RCGR protocol is a combination of robot gait and motor imagery training, we cannot differentiate the effect of the two components on brain changes; a controlled study may clarify this issue. Finally, the robotic gait system proposed here still has a few shortcomings: firstly, the lack of foot contact prevents meaningful podalic somatosensory information for postural control; secondly, it does not provide ankle motion, which would be important both for clinical reasons and for homogeneity with the fMRI task that we can use to study locomotion (as we already mentioned, fMRI tasks suitable for studying gait neural correlates involve ankle flexion): complete weight support entails less compliance. At present, we are working on the design of a new gait orthosis prototype comprising ground contact and an actuated ankle joint.

\section{ACKNOWLEDGMENTS}

This research was supported by the Piedmont Region, under the 2008 Human and Social Sciences grant, pursuant to Region Law no. 4/2006 (Regione Piemonte, bando Scienze Umane e Sociali 2008, L.R. n. 4/2006). The authors would like to thank the patients who participated in the study and their families.

Dobkin, B. H., Firestine, A., West, M., Saremi, K., and Woods, R. (2004). Ankle dorsiflexion as an fMRI paradigm to assay motor control for walking during rehabilitation. $\mathrm{Neu}$ roimage $23,370-381$.

Driskell, J. E., Copper, C. M., and Moran, A. (1994). Does mental practice enhance performance? J. Appl. Psychol. 79, 481-492.

Dunsky, A., Dickstein, R., Ariav, C., Deutsch, J., and Marcovitz, E. (2006). Motor imagery practice in gait rehabilitation of chronic poststroke hemiparesis: four case studies. Int. J. Rehabil. Res. 29, 351-356.

Enzinger, C., Johansen-Berg, H., Dawes, H., Bogdanovic, M., Collett, J., Guy, C., Ropele, S., Kischka, U., Wade, D., Fazekas, F., and Matthews, P. M. (2008). Functional MRI correlates of lower limb function in stroke victims with gait impairment. Stroke 39, 1507-1513.

Forrester, L. W., Wheaton, L. A., and Luft,A. R. (2008). Exercise-mediated locomotor recovery and lower-limb neuroplasticity after stroke. J. Rehabil. Res. Dev. 45, 205-220.
Friston, K. J., Frith, C. D., Liddle, P. F., and Frackowiak, R. S. (1993). Functional connectivity: the principalcomponent analysis of large (PET) data sets. J. Cereb. Blood Flow Metab. $13,5-14$.

Hidler, J., Nichols, D., Pelliccio, M., Brady, K., Campbell, D. D., Kahn, J. H., and Hornby, T. G. (2009). Multicenter randomized clinical trial evaluating the effectiveness of the Lokomat in subacute stroke. Neurorehabil. Neural Repair 23, 5-13.

Holden, M. K., Gill, K. M., and Magliozzi, M. R. (1986). Gait assessment for neurologically impaired patients. Standards for outcome assessment. Phys. Ther. 66, 1530-1539.

Hornby, T. G., Campbell, D. D., Kahn, J. H., Demott, T., Moore, J. L., and Roth, H. R. (2008). Enhanced gait-related improvements after therapist-versus robotic-assisted locomotor training in subjects with chronic stroke: a randomized controlled study. Stroke 39, 1786-1792. 
Husemann, B., Müller, F., Krewer, C., Heller, S., and Koenig, E. (2007). Effects of locomotion training with assistance of a robotdriven gait orthosis in hemiparetic patients after stroke: a randomized controlled pilot study. Stroke 38, 349-354.

Jackson, P. L., Doyon, J., Richards, C. L., and Malouin, F. (2004). The efficacy of combined physical and mental practice in the learning of a footsequence task after stroke: a case report. Neurorehabil. Neural Repair 18, 106-111.

Jackson, P. L., Lafleur, M. F., Malouin, F., Richards, C., and Doyon, J. (2001). Potential role of mental practice using motor imagery in neurologic rehabilitation. Arch. Phys. Med. Rehabil. 82, 1133-1141.

Jezernik, S., Scharer, R., Colombo, G., and Morari, M. (2003). Adaptive robotic rehabilitation of locomotion: a clinical study in spinally injured individuals. Spinal Cord 41, 657-666.

Karni, A., Meyer, G., Jezzard, P., Adams, M. M., Turner, R., and Ungerleider, L. G. (1995). Functional MRI evidence for adult motor cortex plasticity during motor skill learning. Nature 377, 155-158.

Kelly, C., Foxe, J. J., and Garavan, H. (2006). Patterns of normal human brain plasticity after practice and their implications for neurorehabilitation. Arch. Phys. Med. Rehabil. 87, S20-S29.

Lawrence, D. G., and Kuypers, H. G. (1968). The functional organization of the motor system in the monkey. I. The effects of bilateral pyramidal lesions. Brain 91, 1-14.

Li, X., Xia, H., and Guan, T. (2008). "Development of legs rehabilitation exercise system driven by pneumatic muscle actuator," in Proceeding of the 2nd International Conference on Bioinformatics and Biomedical Engineering, Shanghai.

Mahoney, F. I., and Barthel, D. W. (1965). Functional evaluation: the
Barthel index. Md. State Med. J. 14, 61-65.

Malouin, F., and Richards, C. L. (2010). Mental practice for relearning locomotor skills. Phys. Ther. 90, 240-251.

Malouin, F., Richards, C. L., Doyon, J., Desrosiers, J., and Belleville, S. (2004). Training mobility tasks after stroke with combined mental and physical practice: a feasibility study. Neurorehabil. Neural Repair $18,66-75$.

Mayr, A., Kofler, M., Quirbach, E., Matzak, H., Frohlich, K., and Saltuari, L. (2007). Prospective, blinded, randomized crossover study of gait rehabilitation in stroke patients using the Lokomat gait orthosis. Neurorehabil. Neural Repair 21, 307-314.

Mehrholz, J., Werner, C., Kugler, J., and Pohl, M. (2007). Electromechanicalassisted training for walking after stroke. Cochrane Database Syst. Rev. 4, CD006185.

Milton, J., Small, S. L., and Solodkin, A. (2008). Imaging motor imagery: methodological issues related to expertise. Methods 45, 336-341.

Nelles, G. (2004). Cortical reorganization - effects of intensive therapy. Restor. Neurol. Neurosci. 22, 239-244.

Pascual-Leone, A., Grafman, J., and Hallett, M. (1994). Modulation of cortical motor output maps during development of implicit and explicit knowledge. Science 263, 1287-1289.

Phillips, J. P., Sullivan, K. J., Burtner, P. A., Caprihan, A., Provost, B., and Bernitsky-Beddingfield, A. (2007). Ankle dorsiflexion fMRI in children with cerebral palsy undergoing intensive body-weightsupported treadmill training: a pilot study. Dev. Med. Child Neurol. 49, 39-44.

Rossini, P. M., Altamura, C., Ferreri, F., Melgari, J. M., Tecchio, F., Tombini, M., Pasqualetti, P., and Vernieri, F. (2007). Neuroimaging experimental studies on brain plasticity in recovery from stroke. Eura. Medicophys. 43, 241-254.

Sacco, K., Cauda, F., Cerliani, L., Mate, D., Duca, S., and Geminiani, G. C. (2006). Motor imagery of walking following training in locomotor attention. The effect of "the tango lesson." Neuroimage 32, 1441-1449.

Sacco, K., Cauda, F., D'Agata, F. Mate, D., Duca, S., and Geminiani, G. (2009). Reorganization and enhanced functional connectivity of motor areas in repetitive ankle movements after training in locomotor attention. Brain Res. 1297, 124-134.

Sahyoun, C., Floyer-Lea, A., JohansenBerg, H., and Matthews, P. M. (2004). Towards an understanding of gait control: brain activation during the anticipation, preparation and execution of foot movements. $\mathrm{Neu}$ roimage $21,568-575$.

Schwartz, I., Sajin, A., Fisher, I., Neeb, M., Shochina, M., Katz-Leurer, M., and Meiner, Z. (2009). The effectiveness of locomotor therapy using robotic-assisted gait training in subacute stroke patients: a randomized controlled trial. PM R 1, 516-523.

Sirigu, A., Cohen, L., Duhamel, J. R. Pillon, B., Dubois, B., Agid, Y., and Pierrot-Deseilligny, C. (1995). Congruent unilateral impairments for real and imagined hand movements. Neuroreport 6, 997-1001.

Talairach, J., and Tournoux, P. (1988). Co-planar Stereotaxic Atlas of the Human Brain: 3-Dimensional Proportional System: An Approach to Cerebral Imaging. Stuttgart: Thieme.

Waldner, A., Tomelleri, C., and Hesse, S. (2009). Transfer of scientific concepts to clinical practice: recent robot-assisted training studies. Funct. Neurol. 24, 173-177.

Westlake, K. P., and Patten, C. (2009). Pilot study of Lokomat versus manual-assisted treadmill training for locomotor recovery post-stroke. J. Neuroeng. Rehabil. 6, 18.

Winchester, P., McColl, R., Querry, R., Foreman, N., Mosby, J., Tansey, K., and Williamson, J. (2005). Changes in supraspinal activation patterns following robotic locomotor therapy in motor-incomplete spinal cord injury. Neurorehabil. Neural Repair 19, 313-324.

Wirz, M., Zemon, D. H., Rupp, R., Scheel, A., Colombo, G., Dietz, V., and Hornby, T. G. (2005). Effectiveness of automated locomotor training in patients with chronic incomplete spinal cord injury: a multicenter trial. Arch. Phys. Med. Rehabil. 86, 672-680.

Yen, C. L., Wang, R. Y., Liao, K. K., Huang, C. C., and Yang, Y. R. (2008). Gait training induced change in corticomotor excitability in patients with chronic stroke. Neurorehabil. Neural Repair 22, 22-30.

Conflict of Interest Statement: The authors declare that the research was conducted in the absence of any commercial or financial relationships that could be construed as a potential conflict of interest.

Received: 09 September 2011; accepted: 07 November 2011; published online: 23 November 2011.

Citation: Sacco K, Cauda F, D'Agata F, Duca S, Zettin M, Virgilio R, Nascimbeni A, Belforte G, Eula G, Gastaldi L, Appendino $S$ and Geminiani G (2011) A combined robotic and cognitive training for locomotor rehabilitation: evidences of cerebral functional reorganization in two chronic traumatic brain injured patients. Front. Hum. Neurosci. 5:146. doi: 10.3389/fnhum.2011.00146 Copyright (c) 2011 Sacco, Cauda, D'Agata, Duca, Zettin, Virgilio, Nascimbeni, Belforte, Eula, Gastaldi, Appendino and Geminiani. This is an open-access article subject to a non-exclusive license between the authors and Frontiers Media $S A$, which permits use, distribution and reproduction in other forums, provided the original authors and source are credited and other Frontiers conditions are complied with. 